\title{
Excellence and power in the Black physics community
}

\author{
\#BlackInPhysics Week aimed to build community among physicists by celebrating, supporting and increasing the \\ visibility of Black physicists. The week accomplished all of this, and more.
}

\section{Charles D. Brown II and Eileen Gonzales}

\begin{abstract}
n 2020, racial inequities driven by deep-seated anti-Black biases came to the attention of the general public in the United States and around the world, catalysed by the swift and unjust murders of George Floyd, Breonna Taylor, Ahmaud Arbery and too many others. Within academia, issues of racial inequality materialized in the \#BlackInTheIvory outpouring on Twitter, where tens of thousands of Black people described interpersonal and systemic racism in their institutions of higher education. These inequities coupled with the COVID-19 global pandemic that has disproportionately harmed Black people have been powerful sources of both isolation and attacks on belonging. In May 2020, a woman knowingly used the threat of police violence against an innocent Black man who was birdwatching in Central Park, and in response, the social media campaign \#BlackBirdersWeek was created to highlight the multifaceted interests and expertise of Black people. This campaign rippled through the Black scientific community, sparking a desire to use online platforms to address the issues
\end{abstract} faced by Black physicists, and to highlight their many scientific contributions. And so, \#BlackInPhysics Week was born ${ }^{1,2}$.

The event, which took place in the last week of October, was an international initiative primarily focused on building community. We believe that community is critical as it provides an anchor that gives one a foundation and a strong sense of belonging in their craft or discipline. Our aim was to strengthen intergenerational connections between Black physicists, encourage long-lasting collaborations, and further push for the development of supportive environments where current and future Black physicists could thrive. Equally, celebrating Black physicists and highlighting their contributions to the wider community paints a more complete picture of what a physicist looks like. In addition, we aimed to inspire future Black physicists by communicating exciting new findings in the world of physics research.

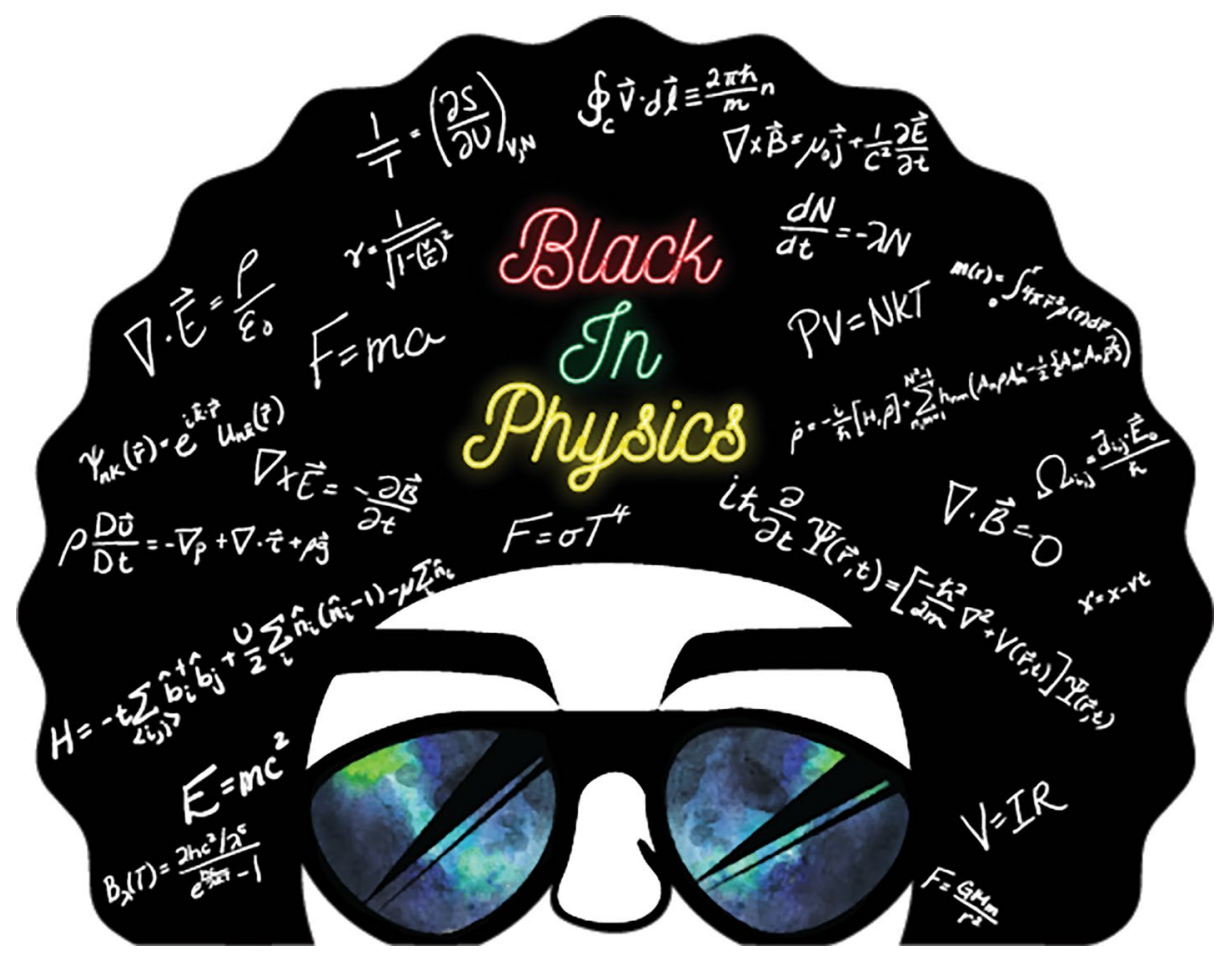

Credit: Courtesy of the \#BlackInPhysics week organizers

\#BlackInPhysics Week helped to make clear that, globally, Black physicists have a substantial stake in physics. Both our past and future scientific contributions make up an important part of the human endeavour to understand the Universe. To promote individual Black physicists' visibility, we prompted a \#BlackInPhysicsRollCall, asking Black physicists to use this hashtag on Twitter to describe both themselves and their research. One can now simply search for the Twitter hashtag \#BlackInPhysicsRollCall and find information about talented Black physicists around the world.

To further our community building efforts and engagement on Twitter, we divided the week into various physics subfields such as physics education research (\#BlackInPER), soft-matter physics (\#BlackInSquishyPhysics) and industry (\#BlackInIndustry). Each day we engaged with the community by initiating discussions around a \#EquationoftheDay and a \#PhysicsPictureoftheDay, highlighting Black physicists in the subfield of the day, and sharing facts about aspects of the subfield. By the week's end, \#BlackInPhysics Week content had been interacted with over two million times.

Our professional and social events helped to reveal a more accurate picture of what a physicist looks like by addressing issues relevant to our multifaceted identities. \#BlackInPhysics Week offered a series of panel discussions that targeted issues relevant to Black physicists, including defining and achieving success as an undergraduate, striving for work-life balance as a graduate student or postdoc, 


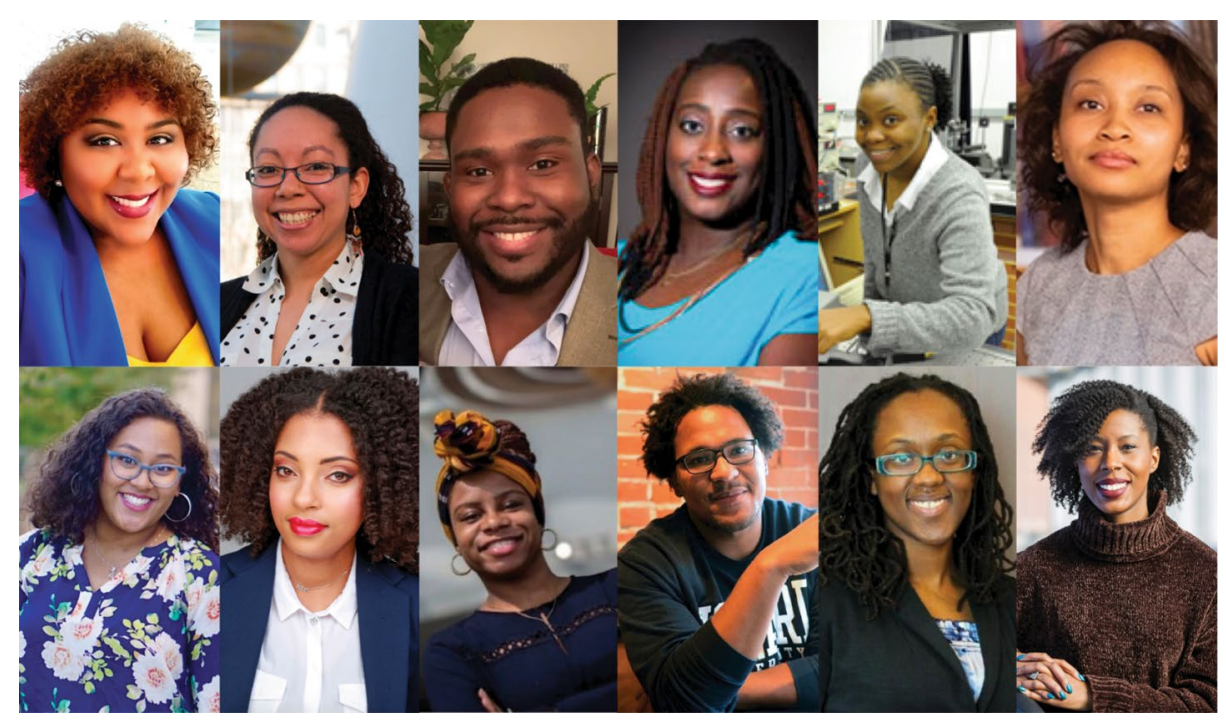

Fig. 1 | \#BlackInPhysics week organizers. Top row (left to right): Dr Jessica Esquivel (lead organizer), Dr Eileen Gonzales (lead organizer), Dr Charles D. Brown II (lead organizer), Dr Vanessa Sanders, Jessica Tucker, LaNell Williams. Bottom row (left to right): Xandria Quichocho, Cheyenne Polius, Ashley Walker, Dr Bryan Ramson, Marika Edwards, Dr Ciara Sivels.

navigating impostor syndrome and mental health on the road to tenure, and forging a non-academic route after college. Our most well-attended panel event was the three-minute thesis competition, where $\mathrm{PhD}$ students showcased their work in an engaging and concise manner. We also set up a virtual job fair, which was free for both jobseekers and employers and attracted over 100 attendees with booths staffed by university physics departments, national labs and companies all looking for excellent Black talent.

The week's social events were effective and multidimensional community-building efforts. The week kicked off with an open-mic night where Black physicists and allies showcased their musical, poetic and comedic talents. Throughout the week, career-stage-specific mixers provided a space to socialize, network and relax with peers and older mentors. Mid-week, an ask-a-scientist panel discussion allowed the general public to engage in conversations with Black physicists conducting cutting-edge research. An all-career-stages mixer at the end of the week provided space for the community to kick back and relax after a long week of fulfilling events. The week's social events ended with a special Halloween virtual murder mystery.

We also commissioned seven essays written by Black physicists on topics related to our identities, co-published by Physics Today ${ }^{3}$ and Physics World ${ }^{4}$. These essays provide important perspectives that are often not given an adequate platform. The authors discuss many topics, including student success, dealing with impostor syndrome and mental health, work-life balance, disability, and the ethics of being Black in physics. Physics Today also highlighted the experiences of Black physicists in their 'Black voices in physics' Q\&A series ${ }^{5}$, where one of our lead organizers shared her experiences.

Increasing diversity in physics has moved to the forefront of discussions on everything from graduate school admissions to hiring faculty. Although these are important topics, the burden placed on Black physicists and physicists of colour to lead initiatives, guide discussions and share stories of our negative experiences is substantial, and this work is often overlooked and vastly undervalued. Reliving such difficult experiences can be traumatic and is often mentally taxing, so we sought to pay our panellists for the wealth of experience and advice that they shared during the week. To this end, \#BlackInPhysics was able to raise enough funds from our sponsors to compensate all our panellists and this is something we want to see normalized for this type of work.

Looking to the future, we remain steadfast in pursuit of our community-building and visibility-building goals. The first tangible result of our efforts is that we now have 250 Black physicists and allies signed up and eager to take part in our future initiatives. This group hails from 12 countries across five continents, spanning school students to academic, government and industry professionals. We hope to continue this movement, making \#BlackInPhysics Week the first of many more to come. With \#BlackInPhysics Week 2021 on the horizon, we would like community input on which events to organize. Community and visibility are crucial for us: we will continue to build space for Black physicists to connect with one another and the broader physics community on our website (blackinphysics.org) and possibly through more frequent quarterly events.

A long-term goal of the \#BlackInPhysics organizers (Fig. 1) is to create a database of Black physicists. This database would not only connect Black physicists with one another but also serve as an invaluable resource for hiring committees and outreach efforts. Many Black physicists are often asked to represent diversity in the field, particularly through school student interview requests and media. To ease this burden, the database would have a correlated collection of news articles, videos and writings for each physicist.

\#BlackInPhysics was not just a week - it was the start of a movement. We aim to be an organization that Black physicists can look to for support, and to make it clear that we are here, pushing for Black physicists' success whether it is inside academia or out there in the world.

Charles D. Brown II ${ }^{1 凶}$ and Eileen Gonzales ${ }^{2,3 凶}$ ${ }^{1}$ Department of Physics, University of California, Berkeley, Berkeley, CA, USA. ${ }^{2}$ Department of Astronomy, Cornell University, Ithaca, NY, USA. ${ }^{3}$ Cornell Center for Astrophysics and Planetary Sciences, Cornell University, Ithaca, NY, USA.

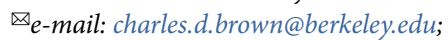
ecg224@cornell.edu

Published online: 4 January 2021 https://doi.org/10.1038/s41567-020-01140-9

\section{References}

1. Brown, C. D. II, Esquivel, J., Gonzales, E., Quichocho, X. \& Polius, C. Meet the organizers of \#BlackInPhysics Week. Physics Today https://doi.org/10.1063/PT.6.4.20201026b (2020).

2. Banks, M. BlackInPhysics week set to celebrate Black physicists. Physics World https://physicsworld.com/a/ blackinphysics-week-set-to-celebrate-black-physicists/ (2020).

3. The organizers of \#BlackInPhysics Week BlackInPhysics Week essay series. Physics Today https://doi.org/10.1063/ PT.6.4.20201026a (2020).

4. \#BlackInPhysics collection. Physics World https://physicsworld $\mathrm{com} / \mathrm{p} /$ collections/blackinphysics/ (2020).

5. Feder, T. Q\&A series: Black voices in physics. Physics Today https://doi.org/10.1063/PT.6.4.20201022a (2020).

Competing interests

The authors declare no competing interests. 